\title{
A case in which a solitary fibrous tumor developed in the retroperitoneal cavity
}

\begin{abstract}
Solitary fibrous tumors (SFTs) were initially reported to be pleural tumors, but they can occur at any site. Herein, we report an unusual case in which a large SFTS developed in the retroperitoneal cavity. A 43-year-old female was referred to our hospital because of abdominal distension. Computed tomography (CT) and magnetic resonance imaging revealed a large retroperitoneal tumor occupying the entire abdominal cavity. In addition, CT showed that the right adrenal artery was the tumor's main feeding artery. At first, we suspected an intra-abdominal tumor, but no conclusive evidence to support this diagnosis was obtained. As there was a possibility of malignancy (and to relieve the patient's symptoms), we performed the surgical resection. A postoperative pathological examination revealed that the tumor was an SFTs derived from the retroperitoneal mesenchyme. The patient has been tumor-free for 5years since the operation. We present a rare case of SFTs that was difficult to differentiate from an adrenal tumor.
\end{abstract}

Keywords: intra-abdominal tumor, retroperitoneal mesenchyme, SFTs, solitary fibrous tumors
Volume 3 Issue 2 - 2017

\author{
Takeshi Morinaga,' Kei Horino,' Shinya \\ Shimada,' Keisuke Morita,' Kenichiro \\ Yamamoto,' Satoshi Ikeshima,' Hideo Baba ${ }^{2}$ \\ 'Department of Surgery, Kumamoto General Hospital, Japan \\ ${ }^{2}$ Department of Gastroenterological Surgery, Kumamoto \\ University, Japan
}

\begin{abstract}
Correspondence: Shinya Shimada, Department of Surgery, Kumamoto General Hospital, Community Health Care Organization (JCHO), 10-10 Tohricho, Yatsushiro, Kumamoto 866-8660, Japan, Tel 81965327। II, Fax 81965322772, Email shimada-shinya@kumamoto.jcho.go.jp
\end{abstract}

Received: June 01, 2017 | Published: June 21, 2017
Abbreviations: MRI, magnetic resonance imaging; CT, computed tomography

\section{Introduction}

SFTSs was described in $1931 .^{1}$ It was a pleural tumor. SFTs was subsequently referred to as solitary mesothelioma, benign fibrous mesothelioma, or localized fibrous tumor of the pleura because of its histological features and the fact that many cases involved the pleura. ${ }^{1-3}$ Previously, it was considered that SFTs are derived from mesothelial or mesenchymal tissues, but recent ultrastructural and immunohistochemical studies have strongly indicated that SFTs originate from the mesenchyme. ${ }^{4,5}$ We now know that SFTs can occur at any site, and reports of extra-thoracic SFTs involving sites such as the mediastinum, ${ }^{6,7}$ pericardium, ${ }^{8,9}$ and retroperitoneum ${ }^{10}$ are becoming increasingly common. However, SFTs are difficult to diagnose due to their rarity. Here, we describe a case in which an SFTs arose in the retroperitoneal cavity and was hard to differentiate from an adrenal tumor.

\section{Case report}

A 43year-old female noticed a mass in her abdomen about 1 month before she was referred to our hospital. The area just above the mass was tender, and the mass extended from the right upper abdomen to the lateral abdomen and was the size of a child's head. No other findings were noted during physical examinations. A laboratory analysis produced almost normal results, and computed tomography $(\mathrm{CT})$ and magnetic resonance imaging (MRI) revealed a large retroperitoneal tumor measuring $20 \times 16 \mathrm{~cm}$ occupying the entire abdominal cavity. The tumor had pushed the surrounding organs, such as the right kidney and liver, outwards (Figure 1). The right adrenal artery was the tumor's main feeding artery, but many other arteries surrounded and fed the tumor (Figure 2). The tumor's interior had a non-uniform structure; some areas exhibited early enhancement, whereas other areas were barely enhanced. There were no signs of distant metastasis or infiltration into the surrounding tissues. From the above findings, we initially suspected an adrenal tumor, but no conclusive diagnostic evidence was obtained. A further laboratory analysis produced the following results: adrenaline, $10 \mathrm{pg} / \mathrm{mL}$; noradrenaline, $189 \mathrm{pg} / \mathrm{mL}$; dopamine, $<5 \mathrm{pg} / \mathrm{mL}$; cortisol, $17.1 \mu \mathrm{g} / \mathrm{d}$; aldosterone, $398 \mathrm{pg} / \mathrm{mL}$; dehydroepiandrosterone sulfate, $56 \mu \mathrm{g} /$ $\mathrm{dL}$; plasma renin activity, $4.7 \mathrm{ng} / \mathrm{mL} / \mathrm{h}$; urinary vanillylmandelic acid, $4.2 \mathrm{mg} /$ day; urinary metanephrine, $0.09 \mathrm{mg} /$ day; urinary normetanephrine, $0.24 \mathrm{mg} /$ day. These findings suggested that the mass was a non-functioning tumor. In addition, no accumulation was seen in the adrenal region on metaiodobenzylguanidine scintigraphy. Finally, we performed a complete surgical resection of the tumor and the right adrenal gland because of the possibility of malignancy and to relieve the patient's symptoms. Macroscopically, the surgical specimen was $20.0 \times 20.0 \times 18.0 \mathrm{~cm}$ in size and weighed $1.8 \mathrm{~kg}$. The mass was surrounded by a fibrous capsule and had not infiltrated into the surrounding tissue. A cross-section of the tumor showed a yellowish fibrous lobulated solid mass and some cysts (Figure 3). A pathological examination revealed that the tumor was composed of spindle-shaped cells, which were arranged in the "patternless pattern" and exhibited varying degrees of cellularity and low mitotic activity [1-2 mitoses per 10 high-power field]. Immunohistochemical staining detected positivity for $\mathrm{CD} 34$ and vimentin. On the other hand, the tumor was negative for c-kit, desmin, smooth muscle antigen (SMA), and S-100, which confirmed the diagnosis of a retroperitoneal SFTs. Based on the surgical findings, we suspected that the tumor was of mesenchymal origin; i.e., that it was derived from the nearby right adrenal gland. The patient did not suffer any complications and was discharged. No further treatments were performed, and 5years have passed without recurrence since the operation. 


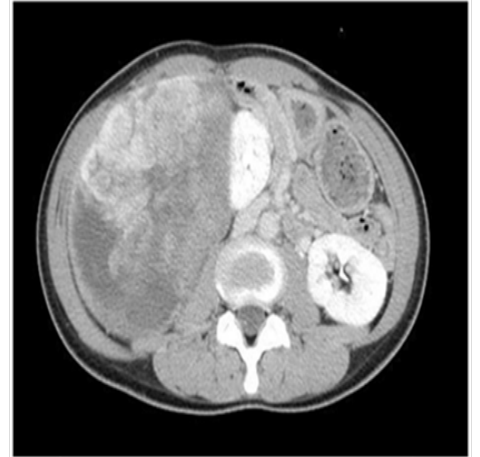

Figure I Macroscopic findings, Axial CT image of the abdomen at the mid abdomen level shows a large retroperitoneal tumor measuring $20 \times 16 \mathrm{~cm}$ was found. It occupied the entire abdominal cavity and had pushed the surrounding organs, such as the right kidney and liver, outwards.

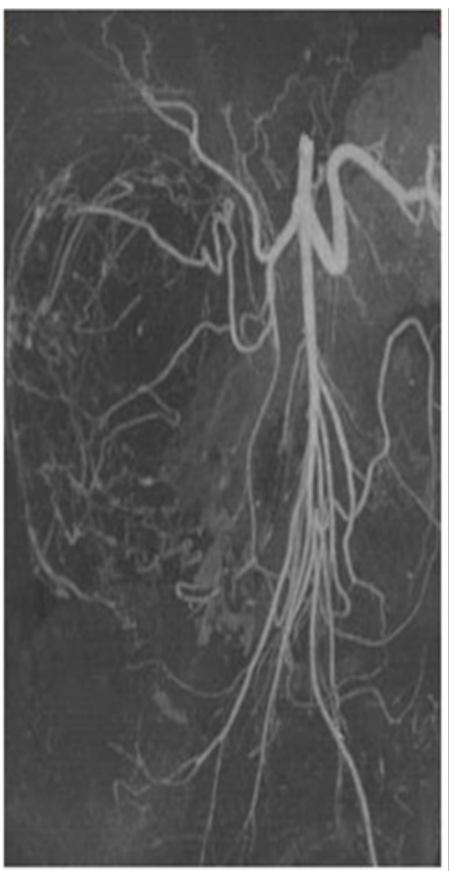

Figure 2 CT findings, CTA of abdominal arteries MIP image shows that the right adrenal artery was the tumor's main feeding artery, but many other arteries surrounded and fed the tumor.

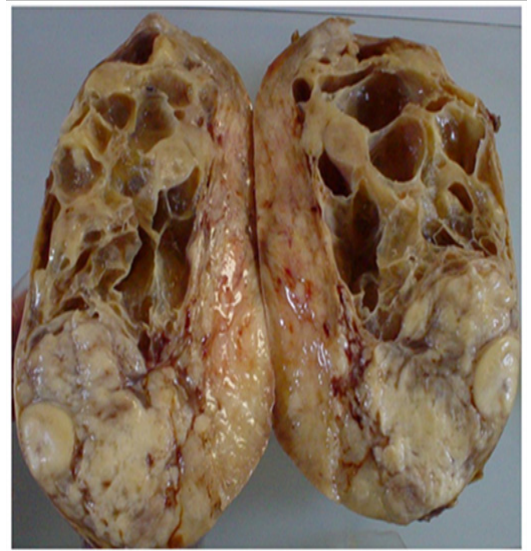

Figure 3 Pathological findings, a cross-section of the tumor showed a yellowish fibrous lobulated solid mass and some cysts.

\section{Discussion}

The first SFTs was described in $1931 .{ }^{1}$ It was a pleural tumor. Incidence of SFTs was reported to be 2.8 cases per 100,000 registrations. ${ }^{2}$ SFTs most commonly occur in individuals in their $60 \mathrm{~s}$ to 70 s and do not exhibit any predilection for either gender. According to previous studies, approximately $30 \%$ of SFTs occur in the extrathoracic region, ${ }^{1}$ but we now know that SFTs can occur any site. In agreement with this, the number of reported cases of extra-thoracic SFTs, including SFTs of the mediastinum, ${ }^{6,7}$ nasal cavity, ${ }^{11}$ liver, ${ }^{12,13}$ kidneys, ${ }^{14}$ pericardium,,${ }^{8,9}$ and retroperitoneum, ${ }^{10}$ is increasing. This report describes a case in which an SFTS arose in the retroperitoneum.

Previously, SFTs were considered to originate from the mesothelium, ${ }^{1-3}$ and thus, were referred to solitary mesothelioma, benign fibrous mesothelioma, or localized fibrous tumors. However, recent ultrastructural and immunohistochemical studies strongly indicated that SFTs originate from the mesenchyme. The histological characteristics of SFTs include the so-called "patternless pattern", which involves a haphazard, storiform arrangement of spindle cells, and a "hemangiopericytoma-like appearance" with prominent vascularity. Both patterns were evident in our case. In addition, immunohistochemical staining of SFTs results in positivity for CD34, $\mathrm{Bcl} 2$, and vimentin and negativity for c-kit, desmin, SMA, and S-100. In our case, CD34 and vimentin staining were positive. On the other hand, c-kit, desmin, SMA, and S-100 staining were negative. These results confirmed the diagnosis of SFTs.

Some cases of extra-thoracic SFTs are found after the detection of hypoglycemia, which is attributed to insulin-like growth factor produced by the tumor (incidence: about $5 \%$ ). ${ }^{15}$ However, many extrathoracic SFTs are encountered during the course of examinations for other disorders, such as stomach ache or dysuria, or during medical check-ups. ${ }^{16}$ Some cases of SFTs in retroperitoneum were found by palpable swelling in abdomen, but a certain case was found by dysuria because ureter runs to bladder in retroperitoneal cavity. ${ }^{17}$ In our case, palpable swelling in abdomen was a trigger to find her SFTs. SFTs have almost no specific symptoms, and thoracic SFTS might have been found first because they are easy to detect on X-ray examinations, whereas extra-thoracic SFTS are difficult to detect until they progress to some extent. It is hard to diagnose SFTs preoperatively because they do not exhibit any specific findings. So, clinicians should be aware that SFTs could occur at any site. The basic treatment for SFTs is resection with sufficient clear margins. Although most SFTs usually have a good prognosis, they have the potential to recur or metastasize after surgery, and so careful follow-up is required after complete resection.

\section{Summary}

In summary, we experienced a rare case in which SFTs developed in the retroperitoneal cavity, which was hard to differentiate from an adrenal tumor. We performed complete surgical resection, and 5years have passed without recurrence since the operation.

\section{Acknowledgements}

None.

\section{Conflict of interest}

Author declares that there is no conflict of interest. 


\section{References}

1. Klempere P, Rabin CB. Primary neoplasm of the pleura: a case report of five cases. Arch Pathol. 1931;11:385-412.

2. Okike N, Bernatz PE, Woolner LB. Localized mesothelioma of the pleura. Benign and malignant variants. J Thorac Cardiovasc Surg. 1978;75(3):363-372.

3. Briselli M, Mark EJ, Dckersin GR. Solitary fibrous tumors of the pleura. Eight new cases and review of 360 cases in the literature. Cancer. 1981;47(11):2678-2689.

4. Suster S, Nascimento AG, Miettinen M, et al. Solitary fibrous tumors of soft tissue. A clinicopathological and immunohistochemical study of 12 cases. Am J Surg Pathol. 1995;19(11):1257-1266.

5. Mentzel T, Bainbridge TC, Katenkamp D. Solitary fibrous tumor: Clinicopathological, immunohistochemical, and ultrastructural analysis of 12 cases arising in soft tissues, nasal cavity and nasopharynx, urinary bladder, and prostate. Virchows Arch. 1997;430:445-453.

6. Weidner N. Solitary fibrous tumor of the mediastinum. Ultrastruct Pathol. 1991;15(4-5):489-492.

7. Goto Y, Sakurada T, Suzuki I, et al. A localized fibrous tumor (mesothelioma) in the mediastinum: report of a case. Surg Today 1997;27(9):871-873.

8. Andreani SM, Tavecchio L, Giardini R, et al. Extrapericardial solitary fibrous tumor of the pericardium. Eur J Cardiothorac Surg. 1998;14(4):98-100.
9. Bortolotti U, Calabro F, Loy M, et al. Giant intrapericardial solitary fibrous tumor. Ann Thorac Surg. 1992;54(6):1219-1220.

10. Piazza R, Blandamura S, Zattoni F, et al. Solitary fibrous tumour of the retroperitoneum mimicking a renal mass. Int Urol Nephrol. 1996;28(6):751-754.

11. Mentzel T, Bainbridge TC, Katenkamp D. Solitary fibrous tumour: clinicopathological, immunohistochemical, and ultrastructural analysis of 12 cases arising in soft tissues, nasal cavity and nasopharynx, urinary bladder and prostate. Virchows Arch. 1997;430:445-453.

12. Moran CA, Ishak KG, Goodman ZD. Solitary fibrous tumor of the liver: a clinicopathologic and immunohistochemical study of nine cases. Ann Diagn Pathol. 1998;2(1):19-24.

13. Fuksbrumer MS, Klimstra D, Panicek DM. Solitary fibrous tumor of the liver: imaging findings. Am J Roentgenol. 2000;175:1683-1687.

14. Gelb AB, Simmons ML, Weidner N. Solitary fibrous tumor involving the renal capsule. Am J Surg Pathol. 1996;20(10):1288-1295.

15. Weiss SW, Goldblum JR. Soft tissue tumors. 4th ed. St Louis: Mosby; 2001.

16. Hasegawa T, Matsuno Y, Shimoda T, et al. Extrathoracic solitary fibrous tumors: their histological variability and potentially aggressive behavior. Hum Pathol. 1999;30(12):1464-1473.

17. Akinori N, Masanori O, Kazumi S, et al. Retroperitoneal Solitary Fibrous Tumor: A Case Report. Hinyokika Kiyo. 2009;55(8):503-507. 